\title{
Neoadjuvant chemotherapy with gemcitabine for pancreatic cancer increases in situ expression of the apoptosis marker M30 and stem cell marker CD44
}

\author{
HIDEHIRO TAJIMA $^{1}$, TETSUO OHTA ${ }^{1}$, HIROHISA KITAGAWA ${ }^{1}$, KOICHI OKAMOTO ${ }^{1}$, SEISHO SAKAI ${ }^{1}$, \\ JUN KINOSHITA $^{1}$, ISAMU MAKINO ${ }^{1}$, HIROYUKI FURUKAWA ${ }^{1}$, HIRONORI HAYASHI ${ }^{1}$, KEISHI NAKAMURA ${ }^{1}$, \\ KATSUNOBU OYAMA ${ }^{1}$, MASAFUMI INOKUCHI ${ }^{1}$, HISATOSHI NAKAGAWARA ${ }^{1}$, HIDETO FUJITA ${ }^{1}$, \\ HIROYUKI TAKAMURA ${ }^{1}$, ITASU NINOMIYA ${ }^{1}$, SACHIO FUSHIDA ${ }^{1}$, TAKASHI TANI ${ }^{1}$, TAKASHI FUJIMURA ${ }^{1}$, \\ SEIKO KITAMURA ${ }^{2}$, HIROKO IKEDA ${ }^{2}$ and KOICHI TSUNEYAMA ${ }^{3}$ \\ ${ }^{1}$ Department of Gastroenterologic Surgery Division of Cancer Medicine, Graduate School of Medicine Science, \\ Kanazawa University; ${ }^{2}$ Division of Pathology, Kanazawa University Hospital, Kanazawa; \\ ${ }^{3}$ Division of Pathology, Toyama University Hospital, Toyama, Japan
}

Received July 2, 2011; Accepted December 23, 2011

DOI: $10.3892 / 01.2012 .657$

\begin{abstract}
We examined the pathological effects of preoperative neoadjuvant chemotherapy (NAC) and the expression of markers of apoptosis, epithelial-to-mesenchymal transition (EMT) and cancer stem cells in resected pancreatic cancer specimens from patients treated with gemcitabine as NAC. Immunohistochemical expression of the apoptosis marker M30, EMT marker Snail and stem cell marker CD44 in surgically resected pancreatic cancer specimens were compared between patients treated (NAC group $n=13$ ) and not treated (control group $n=21$ ) with gemcitabine. In the NAC group, the tumor specimens showed tumor cell injury; however, there was no significant reduction of serosal, retroperitoneal, perineural or vascular invasion, lymph node metastasis or tumor size. The expression frequencies of M30 and CD44 were significantly higher in the NAC group (61.5 and 53.8\%) compared to the control group (9.5 and 14.3\%); however, no significant difference in Snail expression was noted between the two groups (53.8 versus 42.9\%). Gemcitabine induced apoptosis of pancreatic cancer cells in vivo; however, it did not reduce the tumor burden. Moreover, the residual cancer tissues were rich in chemoresistant cancer stem cells. By contrast, marked EMT of cancer cells was observed in the specimens from the groups treated and not treated with gemcitabine.
\end{abstract}

Correspondence to: Dr Hidehiro Tajima, Department of Gastroenterologic Surgery Division of Cancer Medicine, Graduate School of Medicine Science, Kanazawa University, 13-1 Takaramachi, Kanazawa 920-8641, Japan

E-mail: hidetaji@staff.kanazawa-u.ac.jp

Key words: pancreatic cancer, gemcitabine, neoadjuvant chemotherapy, apoptosis, epithelial-to-mesenchymal transition, cancer stem cells

\section{Introduction}

Pancreatic cancer is one of the leading causes of cancer-related mortality worldwide (1). The 5-year survival rate of patients with pancreatic cancer is only approximately 4 to $5 \%$; therefore, the mortality rate is almost on par with the incidence rate (2). Resectability is considered as the most significant prognostic factor $(3,4)$. However, even after curative resection, the 5-year survival rate is only 10 to $25 \%$, seemingly due to the high rates of local recurrence, liver metastasis, lymph node recurrence and peritoneal dissemination (5). Gemcitabine, a pyrimidine analog, was launched in 1996, and has been used as the first-line agent for the treatment of pancreatic cancer (6). Recently, the efficacies of adjuvant and neoadjuvant chemotherapy (NAC) with gemcitabine for pancreatic cancer were reported $(7,8)$.

Chemotherapy and radiotherapy is known to induce apoptotic cell death in malignant tumors (9). In addition, it has recently been reported that anti-cancer treatments may also induce epithelial-to-mesenchymal transition (EMT) in cancer cells, which may play an important role in the aggressive behavior of tumors (10-12). Previous studies in solid tumor cell lines have linked the development of chemoresistance to the occurrence of EMT $(10,13)$. EMT is a key event in the tumor invasion process, whereby epithelial cell layers lose polarity and cell-cell contacts and undergo a marked remodeling of the cytoskeleton (14). Among the hallmarks of EMT are the loss of E-cadherin (14), expression of N-cadherin, the so-called cadherin switch (15), expression of vimentin, a mesenchymal marker, nuclear translocation of $\beta$-catenin, and increased production of transcription factors, including Snail, Twist and Slug (16). Although the precise mechanisms underlying these alterations are unknown, they appear to be linked to alterations in $\beta$-catenin signaling, and E-cadherin functions, and activation of the transcription factors Twist, Slug, and/or Snail $(16,17)$. Shah et al demonstrated an increased number of 
cancer stem cells, which show aggressive behavior and EMT in a gemcitabine-resistant pancreatic cancer cell population (13).

Accumulating evidence suggests that pancreatic stromal cells promote the progression and EMT of pancreatic cancer by increasing cancer cell proliferation and invasion as well as by protecting the cells from radiation- and gemcitabineinduced apoptosis (18). There are a number of reports on the induction of chemoresistance linked to EMT and cancer stem cells in vitro. In stromal-rich tumors, such as scirrhous gastric cancer and pancreatic cancer, the correlation between the tumor and stromal cells is particularly important. However, there is at present no evidence on the expression of EMT and stem cell markers in pancreatic cancer cells following chemotherapy in vivo.

In this study, we comparatively evaluated the expression of the apoptosis marker M30 (caspase-cleaved cytokeratin 18 fragments), EMT marker Snail and stem cell marker CD44 in resected pancreatic cancer specimens from patients administered, and those not administered, preoperative NAC with gemcitabine.

\section{Material and methods}

Patients. Between January 2005 and December 2010 , 34 pancreatic ductal adenocarcinoma patients (21 males and 13 females) underwent surgery at the Department of Gastroenterologic Surgery, Kanazawa University Hospital (Kanazawa, Japan). Among them, 13 patients received preoperative chemotherapy with gemcitabine (NAC group). During the same period, 21 patients did not receive preoperative chemotherapy but underwent surgery (control group). Written informed consent was obtained from all 34 patients prior to their enrollment in the study, and the treatment was undertaken with the approval of the local medical ethics committee.

Pathological specimens. Formalin-fixed and paraffinembedded specimens were retrieved from the surgical pathology files of the Pathology Department of Kanazawa University Hospital. The grading system of Evans et al (19) was used to determine the pathological effects of preoperative chemotherapy. The number of cytological changes and the amount of destruction of the tumor were graded on a scale of I-IV, as follows: grade I, characteristic cytological changes of malignancy are present, but little $(<10 \%)$ or no tumor cell destruction is evident; grade IIa, destruction of $10-50 \%$ of the tumor cells; grade IIb, destruction of $51-90 \%$ of tumor cells; grade III, few $(<10 \%)$ viable-appearing tumor cells are present; grade IIIM, sizable pools of mucin are present; grade IV, no viable tumor cells are present; grade IVM, acellular pools of mucin are present.

Immunohistochemical examination. For immunohistochemical staining, the Dako Envision system, which uses dextran polymers conjugated with horseradish peroxidase (Dako, Carpinteria, CA, USA), was employed to avoid any endogenous biotin contamination. Tissues were fixed with $10 \%$ formaldehyde in phosphate-buffered saline, embedded in paraffin, and cut into 5- $\mu \mathrm{m}$ tissue sections. The sections were deparaffinized in xylene and rehydrated in a graded ethanol series. Endogenous peroxidase was blocked by immersing the
Table I. Patient characteristics.

\begin{tabular}{lccc}
\hline Characteristics & NAC & Control & P-value \\
\hline Patients (n) & 13 & 21 & \\
Gender (n) & & & \\
$\quad$ Male & 7 & 14 & \\
Female & 6 & 7 & N.S. \\
Age (years) & 62.6 & 66.0 & \\
$\quad$ Median & $51-77$ & $52-80$ & N.S. \\
Range & & & \\
Location (n) & 9 & 11 & \\
Head of pancreas & 3 & 10 & N.S. \\
Pancreas body and tail & 3 & & \\
\hline
\end{tabular}

NAC, neoadjuvant chemotherapy; N.S., not significant.

sections in $3 \% \mathrm{H}_{2} \mathrm{O}_{2}$ in $100 \%$ methanol for $20 \mathrm{~min}$ at room temperature. Antigen retrieval was achieved by microwaving sections at $95^{\circ} \mathrm{C}$ for $10 \mathrm{~min}$ in $0.001 \mathrm{M}$ citrate buffer ( $\mathrm{pH} 6.7$ ). After blocking the endogenous peroxidase, the sections were incubated with Protein Block Serum-Free (Dako) at room temperature for $10 \mathrm{~min}$ to block non-specific staining. The sections were then incubated for $2 \mathrm{~h}$ at room temperature with 1:50 diluted mouse monoclonal antibodies against M30 (Peviva, Bromma, Sweden), CD44 (Thermo Fisher Scientific Anatomical Pathology, Fremont, CA, USA) and Snail (Santa Cruz Biotechnology, Santa Cruz, CA, USA). Peroxidase activity was detected with the enzyme substrate 3-amino-9ethyl carbazole. For the negative controls, the sections were incubated with Tris-buffered saline without the primary antibodies. Samples in which at least $10 \%$ of tumor cells were slightly counterstained with Meyer hematoxylin were defined as showing positive staining. The frequency of cells positive for each antibody was reported semi quantitatively as follows: $(-)$, no reaction; $(+)$, mild, with $<30 \%$ of cells positive; $(++)$, moderate, with $30-70 \%$ of cells positive; and (+++), marked, with $>70 \%$ of cells positive. A positive expression was defined as staining of $>30 \%$ of the cancer cells $(++$ or +++$)$.

Statistical analysis. Categorical variables were compared using the Chi-square test. For statistical analysis, $\mathrm{P}$ values were calculated using a two-tailed test. $\mathrm{P}<0.05$ was considered to indicate a statistically significant difference.

\section{Results}

Patient characteristics. Between January 2005 and December 2010, 34 pancreatic ductal adenocarcinoma patients (21 males and 13 females) underwent surgery. Among them, 13 patients, 7 males and 6 females, with an average age of 62.6 years (range, 51-77) underwent preoperative chemotherapy with gemcitabine (NAC group). During the same period, 21 pancreatic cancer patients who did not receive preoperative chemotherapy also underwent surgical resection (control group). These 21 patients comprised 14 male and 7 female patients, with an average age of 66.0 years (range, 52-80). 
Table II. Histopathological characteristics.

\begin{tabular}{lccc}
\hline $\begin{array}{l}\text { Histopathological } \\
\text { characteristics }\end{array}$ & NAC & Control & P-value \\
\hline $\begin{array}{l}\text { Tumor size (mm) } \\
\text { Average }\end{array}$ & 30.1 & 30.6 & \\
Range & $16-55$ & $17-53$ & N.S. \\
Serosal invasion & $53.8 \%$ & $40.0 \%$ & N.S. \\
Retroperitoneal invasion & $84.6 \%$ & $61.9 \%$ & N.S. \\
Vascular invasion & $84.6 \%$ & $90.5 \%$ & N.S. \\
Lymph node metastasis & $76.9 \%$ & $57.1 \%$ & N.S. \\
Perineural invasion & $100.0 \%$ & $90.5 \%$ & N.S. \\
\hline
\end{tabular}

NAC, neoadjuvant chemotherapy; N.S., not significant.

Table III. Expression of M30, CD44 and Snail.

\begin{tabular}{lccc}
\hline Markers & NAC & Control & P-value \\
\hline M30 & 5 & 19 & \\
$\quad$ - or + & 8 & 2 & $<0.05$ \\
$\quad++$ or +++ & & 18 & \\
CD44 & 6 & 3 & 0.02 \\
$\quad$ - or + & 7 & 12 & \\
++ or +++ & & 9 & N.S. \\
Snail & 6 & & \\
- or + & 7 & & \\
++ or +++ & & & \\
\hline NAC, neoadjuvant chemotherapy. & & \\
\hline
\end{tabular}

The patient and tumor characteristics are shown in Table I. There were no significant differences in these characteristics between the two treatment groups.

Histopathological characteristics and pathological response. The histopathological characteristics of the tumors in each group are shown in Table II. Nno significant differences were found with respect to the tumor size, invasion of the serosa, retroperitoneum, vessel or nerve, or lymph node metastasis between the NAC group and the control group. In the NAC group, the tumor specimens showed evidence of tumor cell injury, although, none of the patients exhibited a complete pathological response. The treatment effect was judged by Evans grading to be grade IIa in 11 patients and grade IIb in 2 patients.

Immunohistochemical examination. Approximately 34 surgically resected specimens of pancreatic ductal adenocarcinoma were immunohistochemically examined for M30, CD44 and Snail expression (Table III).

The apoptosis marker M30 was mainly expressed in the nuclei of the cancer cells (Fig. 1). A positive M30 expression was found in 8 of the 13 cases $(61.5 \%)$ of the NAC group and in 2 of the 21 cases $(9.5 \%)$ of the control group; the differ-

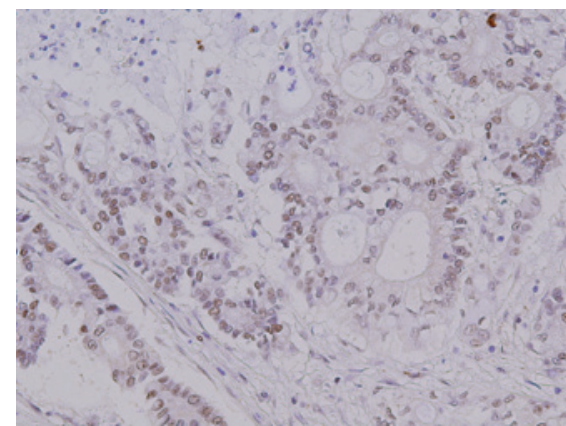

Figure 1. Immunohistochemistry for M30, a marker of apoptosis, in pancreatic cancer tissue. M30 staining was mainly found in the nuclei of the cancer cells. The expression frequency of M30 was significantly higher in the NAC group than that in the control group. NAC, neoadjuvant chemotherapy

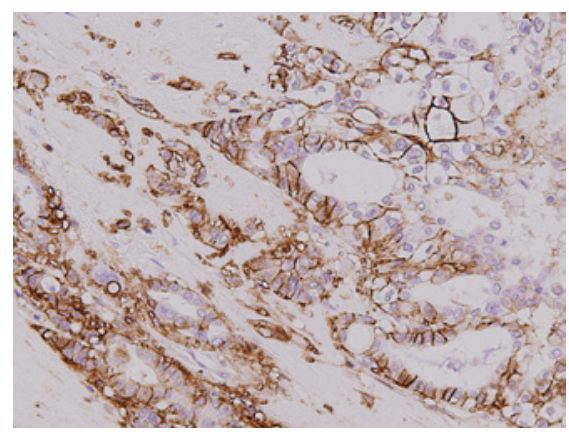

Figure 2. Immunohistochemistry for CD44, a cancer stem cell marker, in pancreatic cancer tissue. CD44 staining was mainly found in the cell surface and cytoplasm in a proportion of cancer cells. A significantly higher frequency of CD44 expression was observed in the NAC group than in the control group. NAC, neoadjuvant chemotherapy.

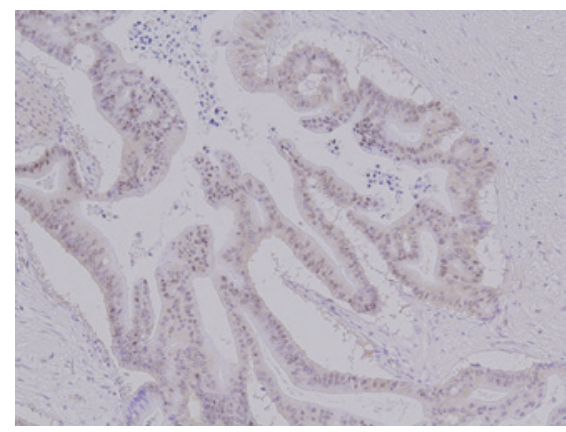

Figure 3. Immunohistochemistry for Snail, a marker of EMT, in pancreatic cancer tissue. Snail was found in the nuclei and cytoplasm of the cancer cells. There was no significant difference in the expression frequency of Snail between the NAC group and the control group. EMT, epithelial-to-mesenchymal transition; NAC, neoadjuvant chemotherapy.

ence between the two groups was statistically significant $(\mathrm{P}=0.002)$.

The pancreatic cancer stem cell marker CD44 was mainly expressed in the cell surface and cytoplasm of a proportion of cancer cells (Fig. 2). A positive CD44 expression was found in 7 of the 13 cases $(53.8 \%)$ in the NAC group and in 3 of the 21 cases $(14.3 \%)$ in the control group; the difference between the two groups was significant $(\mathrm{P}=0.02)$. 
The EMT marker Snail was mainly expressed in the nuclei and cytoplasm of the cancer cells (Fig. 3). A positive Snail expression was observed in 7 of the 13 cases $(53.8 \%)$ in the NAC group, and in 9 of the 21 cases (42.9\%) in the control group; the difference between two groups was not statistically significant. We also examined the expression of other EMT markers such as E-cadherin, N-cadherin and vimentin. However, no significant differences were found in the frequency of expression of these markers between the NAC group and the control group (data not shown).

\section{Discussion}

Despite the rapid advances in diagnostic and surgical techniques, the prognosis of pancreatic cancer remains dismal. Pancreatic ductal adenocarcinoma is characterized by its high malignant potential, showing rapid progression, early metastasis, and limited response to chemotherapy and radiotherapy (18). The efficacies of adjuvant and neoadjuvant chemotherapy with gemcitabine for pancreatic cancer $(7,8)$ and the induction of apoptotic cell death in vitro by chemotherapy and radiotherapy in malignant tumors (9) were reported. Caspase-mediated cleavage of the cytokeratin 18 (CK18) contributes to the degradation of the intracellular cytoskeleton if epithelial cells undergo apoptosis. Recently, plasma caspase-cleaved CK18 fragments (M30) have been reported as specific apoptosis markers and have important clinical biomarker utility (20). In this study, all tumor specimens showed evidence of tumor cell injury following preoperative gemcitabine chemotherapy, and significantly higher expression levels of the apoptosis marker M30 were detected in the NAC group as compared with those in the control group. This finding suggests that gemcitabine induces apoptosis in pancreatic cancer in vivo.

By contrast, gemcitabine-resistant pancreatic tumor cells are more invasive, and show increased migratory potential and increased expression levels of the stem cell marker CD44 (21). It has been reported that chemoradiation-resistant pancreatic cancer cells are similar to cancer stem cells and undergo EMT, suggesting a possible link between chemoradiation resistance-induced EMT and generation of cancer stem cells (13). Moreover, cancer cells showing expression of the stem cell marker CD44 have been reported to be responsible for gemcitabine resistance of pancreatic cancer (22). In this study, the NAC group showed a significantly higher expression frequency of CD44 than the control group. In vivo pancreatic cancers resistant to gemcitabine are rich in cancer stem cells. In cases in which chemotherapy was effective (grade IIb), the majority of the residual tumor cells were found to be positive for CD44 expression.

The interaction between pancreatic cancer cells and stromal cells, such as pancreatic stellate cells and myofibroblasts, is receiving increasing attention. Accumulating evidence suggests that pancreatic stellate cells promote the EMT of cancer cells and the progression of pancreatic cancer by increasing cancer cell proliferation/invasion and by protecting them from radiation- and gemcitabine-induced apoptosis $(18,23)$. Such evidence may explain the limited response of pancreatic cancer in vivo to chemotherapy and radiotherapy. In the present study, the EMT marker Snail was detected at the same level in the NAC and control groups. It has been reported that Snail expression in pancreatic cancer is observed in approximately $36 \%$ of the cells, and that over-expression of Snail increases chemoresistance (24). These results suggest that EMT is induced in pancreatic cancer cells, irrespective of whether the patient receives chemotherapy or not, and the interaction between cancer cells and stromal cells assumes a crucial role in pancreatic cancer.

In the NAC group, the tumor specimens showed evidence of tumor cell injury; however, there were no cases showing a complete pathological response. Furthermore, preoperative chemotherapy with gemcitabine did not result in any significant reduction of serosal/retroperitoneal/perineural/ vascular invasion or lymph node metastases. These results indicate that preoperative chemotherapy for pancreatic cancer with gemcitabine is somewhat effective, but that it does not reduce the tumor burden or the required extent of resection. Therefore, chemoradiotherapy is frequently required for the treatment of unresectable pancreatic cancer (25). Findings of a randomized clinical trial demonstrated that preoperative chemo-radiotherapy is effective for the local control of pancreatic cancer, but that additional radiotherapy tended to increase distant metastasis (26). Moreover, it is reported that several anti-cancer treatments are capable of inducing EMT in cancer tissues (10-12).

In conclusion, preoperative chemotherapy with gemcitabine is effective for pancreatic cancer in vivo. However, this therapy does not result in any decrease of the tumor burden in tumors rich in cancer stem cells or in the required extent of surgical resection. Therefore, counter measures for cancer stem cells and EMT induced by stromal cells are important in pancreatic cancer treatment.

\section{Refeences}

1. Parkin DM, Bray F, Ferlay J and Pisani P: Estimating the world cancer burden: Globocan 2000. Int J Cancer 94: 153-156, 2001

2. Jemal A, Siegel R, Xu J and Ward E: Cancer statistics. CA Cancer J Clin 58: 71-96, 2008.

3. Conlon KC, Klimstra DS, Brennan MF: Long-term survival after curative resection for pancreatic ductal adenocarcinoma. Clinico-pathologic analysis of 5-year survivors. Ann Surg 223: 273-279, 1996.

4. Matsuno S, Egawa S, Fukuyama S, et al: Pancreatic cancer registry in Japan: 20 years of experience. Pancreas 28: 219-230, 2004.

5. Kayahara M, Nagakawa T, Ueno K, et al: An evaluation of radical resection for pancreatic cancer based on the mode of recurrence as determined by autopsy and diagnostic imaging. Cancer 72 : 2118-2123, 1993.

6. Burris HA, Moore MJ, Andersen J, et al: Improvements in survival and clinical benefit with gemcitabine as first-line therapy for patients with advanced pancreatic cancer: a randomized trial. J Clin Oncol 15: 2403-2413, 1997.

7. Ottle H, Post S, Neuhaus P, et al: Adjuvant chemotherapy with gemcitabine vs observation in patients undergoing curative-intent resection of pancreatic cancer: a randomized controlled trial. JAMA 297: 267-277, 2007.

8. Golcher H, Brunner T, Grabenbauer G, et al: Preoperative chemo-radiation in adenocarcinoma of the pancreas. A single centre experience advocating a new treatment strategy. EJSO 34: 756-764, 2008

9. Goses MJ, Dressen RC, Rutten HJ, et al: Prepoerative radio-chemotherapy is successful also in patients with locally advanced rectal cancer who have intrinsically high apoptotic tumors. Ann Oncol 19: 2026-2032, 2008.

10. Yang AD, Fan F, Camp ER, et al: Chronic oxaliplatin resistance induces epithelial-mesenchymal transition in colorectal cancer cell lines. Clin Cancer Res 12: 4147-4153, 2006. 
11. Tsukamoto H, Shibata K, Kajiyama H, et al: Irradiation-induced epithelial-mesencymal transition (EMT) related to invasive potential in endometrial carcinoma cells. Gynecol Oncol 107: 500-504, 2007.

12. Tajima H, Ohta T, Shoji Y, et al: Expression of epithelial-mesenchymal transition markers in locally recurrent hepatocellular carcinoma after radiofrequency ablation. Exp Therap Med 1: 347-350, 2010

13. Shah AN, Summy JM, Zhang J, Park SI, Darikh NU and Gallick GE: Development and characterization of gemcitabine-resistant pancreatic tumor cells. Ann Surg Oncol 14: 3629-3637, 2007.

14. Yang J, Mani SA, Donaher JL, et al: Twist, a master regulator of morphogenesis, plays an essential role in tumor metastasis. Cell 117: 927-939, 2004.

15. Hay ED and Zuk A: Transformations between epithelium and mesenchyme: normal, pathological and experimentally induced. Am J Kidney Dis 26: 678-690, 1995.

16. Thiery JP: Epithelial-mesenchymal transitions in tumor progression. Nat Rev Cancer 2: 442-454, 2002.

17. Thiery JP and Sleeman JP: Complex networks orchestrate epithelial mesenchymal transitions. Nat Rev Mol Cell Biol 7: 131-142, 2006.

18. Kikuta K, Masamune A, Watanabe T, et al: Pancreatic stellate cells promote epithelial-mesenchymal transition in pancreatic cancer cells. Biochem Biophys Res Commun 403: 380-384, 2010.

19. Evans DB, Rich TA, Byrd DR, et al: Preoperative chemoradiation and pancreaticoduodenectomy for adenocarcinoma of the pancreas. Arch Surg 127: 1335-1339, 1992.
20. Dive C, Smith RA, Garner E, et al: Considerations for the use of plasma cytokeratin 18 as a biomarker in pancreatic cancer. $\mathrm{Br} \mathbf{J}$ Cancer 102: 577-582, 2010.

21. Du Z, Qin R, Wei C, et al: Pancreatic cancer cells resistant to chemoradiotherapy rich in 'stem-cell-like' tumor cells. Dig Dis Sci 10: 741-750, 2010.

22. Hong SP, Wen J, Bang S, Park S and Song SY: CD44-positive cells are responsible for gemcitabine resistance in pancreatic cancer cells. Int J Cancer 125: 2323-2331, 2009.

23. Müerköster SS, Werbing V, Koch D, et al: Role of myofibroblasts in innate chemoresistance of pancreatic carcinoma - epigenetic downregulation of caspase. Int J Cancer 123: 1751-1760, 2008.

24. Yin T, Wang C, Liu T, Zhao G, Zha Y and Yang M: Expression of Snail in pancreatic cancer promotes metastasis and chemoresistsance. J Surg Res 141: 196-203, 2007.

25. Cauffert B, Mornex F, Bonnetain F, et al: Phase III trial comparing intensive induction chemoradiotherapy (60 Gy, infusional 5-FU and intermittent cicplatin) followed by maintenance gemcitabine with gemcitabine alone for locally advanced unresectable pancreatic cancer. Definitive results of the 2000-01 FFCD/SFRO study. Ann Oncol 19: 1592-1599, 2008.

26. Van Laethem JL, Hammel P, Mornex F, et al: Adjuvant gemcitabine alone versus gemcitabine-based chemoradiotherapy after curative resection for pancreatic cancer: a randomized EORTC-40013-22012 /FFCD-9203/GERCOR phase II study. J Clin Oncol 28: 4450-4456, 2010. 